\title{
Are Trekking Poles Helping or Hindering Your Hiking Experience? A Review
}

\author{
Ashley L. Hawke, MS; Randall L. Jensen, PhD \\ School of Health and Human Performance, Northern Michigan University, Marquette, MI
}

\begin{abstract}
Hiking is a common recreational activity that provides numerous health benefits, such as reduced risk of heart disease, reduced blood pressure, and improved cardiorespiratory fitness. The use of specifically designed trekking poles has become popular among participants seeking to alleviate sore knees and increase balance and stability while walking. This review provides an overview of physiologic and biomechanical responses elicited when trekking poles are used during outdoor activities, such as hiking or Nordic walking, and discusses the clinical implications of the use of trekking poles. Google Scholar, PubMed, and ScienceDirect databases, as well as university library catalogues, were searched for literature published between 1980 and 2019. The keywords used to search the literature were hiking poles, trekking poles, and Nordic walking and their combination with physiological responses, ground reaction forces, joint forces, spatiotemporal parameters, kinematics, electromyography, and/or balance. The related topics included the academic disciplines of biomechanics, sports science, and wilderness medicine. Reference lists of located studies were also reviewed for additional sources. During free, unloaded walking, users should compare the cost and benefit of using poles: Trekking poles decrease lower extremity loading and forces but increase cardiovascular demand. When carrying a large external load, trekking poles may offer benefit by decreasing lower extremity muscle activity and increasing balance and stability.
\end{abstract}

Keywords: Nordic walking, downhill, uphill, walking aids

\section{Introduction}

Hiking is a popular recreational activity that is both accessible and cost-effective. It provides numerous health benefits, such as reduced blood pressure, risk of heart disease, and improved cardiorespiratory fitness. ${ }^{1}$ Depending on personal preference, physical condition, and experience, hiking can range from a leisurely, low-intensity activity to a strenuous, high-intensity workout. Owing to the physically demanding nature of the activity, hiking may increase injury risk, with the most common being overuse and traumatic injuries (ie, fractures and sprains). ${ }^{2}$ These injuries account for $75 \%$ of injuries to the lower extremities. ${ }^{2}$ When backpacking, hikers typically carry a large load, consisting of equipment such as water, shelter, clothing, food,

Corresponding author: Ashley L. Hawke, MS, School of Health and Human Performance, Northern Michigan University, 1401 Presque Isle Ave, Marquette, MI 49855; e-mail: asvansum@nmu.edu.

Submitted for publication November 2019.

Accepted for publication June 2020. and miscellaneous items. Increased load carriage has been found to result in kinematic and inertial consequences, energetic effects, and local muscle fatigue. ${ }^{3}$ A popular method to combat this increased stress is use of poles to help avoid potential injuries. ${ }^{4}$

The use of trekking poles originated in Finland, when a sports institute and a ski pole company developed specialized high-performance poles that helped refine a technique that was later termed Nordic walking. ${ }^{5}$ Because Nordic walking is a time-efficient, low-stress, yet challenging total body workout, it has rapidly spread worldwide. ${ }^{4,5}$ Nordic walking's popularity has grown because of its active use of the upper body. Several anecdotal claims have been made by those who strongly support use of trekking poles, including that poles alleviate sore knees, hyperflexed posture, and swollen feet and improve balance during outdoor activities. ${ }^{4,6,7}$ Hiking poles, Nordic walking poles, and trekking poles are essentially the same piece of equipment used during outdoor activities; however, for consistency, they will be referred to as trekking poles for the remainder of this review. 
Table 1. Summary of physiological and subjective responses to using trekking poles compared to free walking

\begin{tabular}{|c|c|c|c|c|c|c|c|}
\hline Physiologic and subjective variables & $\dot{V}_{E}$ & $\dot{V} \mathrm{O}_{2}$ & $E E$ & $H R$ & MET & $R P E$ & DOMS \\
\hline Knight and Caldwell, $2000^{4}$ & - & $\leftrightarrow$ & - & $\uparrow$ & - & $\downarrow$ & - \\
\hline Saunders et al, $2008^{16}$ & $\uparrow$ & $\uparrow$ & - & $\uparrow$ & - & $\leftrightarrow$ & - \\
\hline Hansen and Smith, $2009^{12}$ & $\uparrow$ & $\uparrow$ & $\uparrow$ & - & $\uparrow$ & $\leftrightarrow$ & - \\
\hline Howatson et al, $2010^{8}$ & - & - & - & $\leftrightarrow$ & - & $\downarrow$ & $\downarrow$ \\
\hline Pellegrini et al, $2015^{12}$ & - & $\uparrow$ & $\uparrow$ & - & - & - & - \\
\hline Revord et al, $2016^{18}$ & - & - & - & $\leftrightarrow$ & - & $\leftrightarrow$ & - \\
\hline Grainer et al, $2017^{14}$ & - & - & $\uparrow$ & $\uparrow$ & - & $\leftrightarrow$ & - \\
\hline
\end{tabular}

$\dot{\mathrm{V}}_{\mathrm{E}}$, minute ventilation; $\dot{\mathrm{VO}}_{2}$, oxygen uptake; EE, energy expenditure; HR, heart rate; MET, metabolic equivalent of task; RPE, rating of perceived exertion; DOMS, delayed onset muscle soreness.

Use of trekking poles may elicit both positive and negative consequences. Benefits of using poles include improved balance and decreased load on the lower extremities, as well as lower ratings of perceived exertion (RPE) and fewer instances of delayed onset muscle soreness (DOMS). ${ }^{4,6,8-11}$ Negative effects of using trekking poles include increased oxygen uptake $\left(\mathrm{V}_{2}\right)$ and energy expenditure (EE), as well as increased loading of the upper extremities. ${ }^{12-16}$ The changes in physiologic, subjective, and biomechanical responses will be discussed in this review, as well as specific populations' responses to using trekking poles, such as those with low back pain, Parkinson's disease, and elderly and obese populations.

\section{Methods}

Google Scholar, PubMed, and ScienceDirect databases, as well as university library catalogues, were searched for literature published between 1980 and 2019. The terms used to search and select relevant literature were hiking poles, trekking poles, and Nordic walking and their combination with physiological responses, ground reaction forces, joint forces, spatiotemporal parameters, kinematics, electromyography, and/or balance. Literature regarding clinical populations, such as elderly and obese populations and those with Parkinson's disease and low back pain, were selected because of their recurrence when searching for relevant publications. Related topics included the academic disciplines of biomechanics, sports science, and wilderness medicine. Reference lists of located studies were also reviewed for additional sources.

\section{Discussion}

\section{PHYSIOLOGIC RESPONSES}

The effects of trekking poles on physiologic responses during level, uphill, and downhill walking have been investigated in hiking and Nordic walking (Table 1). 4,12,14,16 Researchers have concluded that use of trekking poles may elicit unintended consequences, such as increased physiologic stress and cardiovascular demand, resulting in the activity being more strenuous than anticipated. When an individual uses trekking poles, ventilation, $\dot{\mathrm{V}}_{2}, \mathrm{EE}$, and heart rate (HR) may increase as a result of the engagement of the muscle mass of the upper body, as well as increased load carriage. ${ }^{4,12,14,16}$ It has been suggested that when an individual uses trekking poles, HR increases owing to increased delivery of blood to upper extremities, rather than a response to increased overall workload. ${ }^{4}$ Although use of poles increases upper body involvement, therefore leading to increased total $\mathrm{EE}$, it is unclear how lower body EE is affected; further research in this area should be conducted.

Although it has been shown that the use of trekking poles increases cardiovascular demands owing to the involvement of the upper extremities, it is unclear how these demands change under different surface conditions. Past research has investigated the metabolic costs of trekking pole use in a controlled environment (ie, treadmill walking in a laboratory), with few studies conducted in an actual outdoor environment. $\dot{\mathrm{VO}}_{2}$ was found to increase when walking on grass compared to artificial track and concrete. This was attributed to the fact that when walking on softer surfaces, such as grass, there is an increase in external mechanical work done by the participant, leading to an increased $\dot{\mathrm{V}}_{2} .{ }^{17}$

Several studies have examined subjective responses to the use of trekking poles, such as DOMS or RPE (Table 1). ${ }^{4,8,12,14,16,18}$ What little research has been done on RPE has mainly focused on ascending grades. During 3 different sections of an ascent of the highest mountain in the United Kingdom, RPE was lower when using trekking poles compared to a no-pole condition. ${ }^{8}$ However, there were no differences in RPE during the descent. ${ }^{8}$ Similarly, during a 60-min uphill backpacking task, RPE decreased when using trekking poles. ${ }^{4}$ Despite an increase in cardiovascular demand with the use of poles due to the involvement of the upper body, participants' RPE was lower when they used poles. ${ }^{4,8}$ 
Table 2. Summary of kinematic responses (ie, spatiotemporal measures) when trekking poles are used compared to no poles

\begin{tabular}{|c|c|c|c|c|c|}
\hline Kinematic variables & Step frequency & Step length & Stride frequency & Stride length & Walking speed \\
\hline Knight and Caldwell, $2000^{4}$ & - & - & $\uparrow$ & $\uparrow$ & - \\
\hline Willson et al, $2001^{19}$ & - & - & - & $\uparrow$ & $\uparrow$ \\
\hline Abendroth-Smith et al, $2008^{9}$ & - & - & - & $\uparrow$ & $\uparrow$ \\
\hline Park et al, $2015^{7}$ & - & $\uparrow$ & - & $\uparrow$ & $\uparrow$ \\
\hline Monteiro et al, $2016^{25}$ & - & - & - & - & $\uparrow$ \\
\hline Grainer et al, $2017^{14}$ & $\downarrow$ & - & - & $\uparrow$ & $\uparrow$ \\
\hline
\end{tabular}

The effects of trekking poles on muscle damage and function after mountain descent have also been investigated. ${ }^{8}$ DOMS was examined in both the trekkingpole and no-pole groups: prehike, immediately posthike, and 24,48 , and $72 \mathrm{~h}$ posthike. At $24 \mathrm{~h}$ and $48 \mathrm{~h}$ posthike, DOMS ratings were lower in the trekking-pole group, leading to the conclusion that trekking poles had a greater effect in attenuating muscle damage during descent. ${ }^{8}$

The use of trekking poles may increase cardiovascular demand owing to the increased involvement of the upper extremities. Furthermore, decreases in RPE with the use of poles, despite increased cardiovascular demand, may be important for those looking to incorporate more of a fullbody workout into their outdoor excursions. These findings may be beneficial for those looking to engage in a full-body workout because users can reap the benefits of a high-intensity workout without feeling as though they are overexerting themselves. Conversely, these findings may be important for those who may lack physical conditioning: Poles may make the activity more strenuous than anticipated, leading to greater physiologic stress. Furthermore, those with lower extremity pain may consider the addition of poles for outdoor excursions because use of poles may help alleviate the discomfort that often follows hiking (ie, DOMS), especially during descents or downhill activities.

\section{KINEMATIC AND KINETIC RESPONSES}

In addition to physiologic variables, kinematic variables may be altered with use of poles, specifically spatiotemporal parameters such as step frequency, step length, stride frequency, and walking speed (Table 2). ${ }^{4,7,14,19}$ When walking with a load, use of poles allows individuals to adopt a more normal walking pattern, including a longer stride length and a less flexed knee at heel strike. ${ }^{4}$ As previously shown in multiple studies, trekking poles alter spatiotemporal parameters (ie, increased stride length and frequency), which appears to be advantageous. $4,7,14,19$ Individuals with diseases that affect their gait may benefit from using trekking poles; the use of poles has been shown to improve functional capabilities and allow users to adopt a more normal walking pattern. ${ }^{4,7,14,19}$
Research has also explored kinetics during downhill walking, comparing ground reaction forces (GRF) - the forces between the individual and the ground that are related to load carriage-and forces on lower extremity joints, joint loading forces, and joint moments with and without trekking poles (Table 3). ${ }^{6,7,11,19,20}$ When trekking poles are used, vertical GRF and joint loading of the lower extremities may be reduced by up to $12 \%$ and $16 \%$, respectively. ${ }^{11}$ This may be due to weight dispersion across the upper and lower limbs, with more weight being transferred to the upper extremities and poles. In addition, during walking, forces are likely applied to the poles rather than through the lower extremities. ${ }^{6,7,11,19}$ Use of poles has also been found to result in a more forward trunk lean, leading to a smaller knee moment arm and decreased forces at the knee. ${ }^{11}$

The use of trekking poles has been shown to reduce peak net joint moments in the lower extremities during downhill hiking, both with and without external loads. ${ }^{6}$ Although reductions per impact were rather small, an example could be made from an average 7-km day hike of common hiking trails in 4 national parks. ${ }^{6}$ During downhill walking, the average stride length has been reported to be between $1.25 \mathrm{~m}$ and $1.5 \mathrm{~m} .^{21}$ During a $7-\mathrm{km}$ day hike at an average stride length of $1.4 \mathrm{~m}$, the hiker would experience approximately 5200 impacts $^{6}$; thus, the overall implication of joint moment reductions when poles are implemented would be substantial. In addition, the reductions in the number of impacts, as well as joint moments, may lead to fewer overuse injuries at the muscular level. ${ }^{6}$ Previous research has studied the effects of pole use during downhill and level walking $^{6,7,11,19}$; however, because most trails contain downhill, uphill, and level sections, data must be extrapolated to uphill walking.

In addition to use during downhill walking, use of trekking poles during level walking has been investigated. When walking on level ground, average vertical GRF, compressive knee joint reaction force, and braking impulse all decreased when using poles. ${ }^{19}$ Individuals are also able to walk at a faster speed with poles. Even at a faster speed, lower extremity stress may be reduced with the addition of trekking poles. ${ }^{19}$ It has been proposed that activation of 
Table 3. Summary of kinetic responses when trekking poles are used compared to free walking

\begin{tabular}{lccc}
\hline Kinematic variables & GRF & $\begin{array}{l}\text { Joint } \\
\text { moments }\end{array}$ & $\begin{array}{c}\text { Joint } \\
\text { power }\end{array}$ \\
\hline Schwameder et al, 1999 & $\downarrow$ & $\downarrow$ & - \\
Willson et al, 2001 $^{19}$ & $\uparrow$ & - & - \\
Bohne et al, 2007 & $\uparrow$ & $\downarrow$ & $\downarrow$ \\
Park et al, 2015 & - & - & - \\
\hline
\end{tabular}

GRF, ground reaction force.

the upper extremities when using poles is more a mechanism for balance control than an active contributor to forward propulsion. ${ }^{17}$

Use of trekking poles may decrease lower extremity joint loading during downhill and level walking. This may be especially beneficial for those with knee pain because poles may help alleviate some of the forces exerted on the lower extremities during outdoor hiking activities.

\section{MUSCLE ACTIVITY}

Several studies have examined electromyographic responses during use of trekking poles (Table 4). ${ }^{4,13,15}$ During a treadmill walking task using trekking poles, electromyography showed an increase in upper extremity muscle activation (biceps brachii, triceps, deltoid, latissimus dorsi) with no difference in activation of lower extremity muscles (rectus femoris, biceps femoris, tibialis anterior, and gastrocnemius). ${ }^{16,17}$

Upper extremity muscle activation during loaded uphill treadmill walking with trekking poles was increased, with an almost $300 \%$ increase in the triceps brachii. ${ }^{4}$ When carrying an expedition pack, lower extremity muscle activation decreased, including in the biceps femoris, vastus lateralis, rectus femoris, gastrocnemius, and soleus. ${ }^{4}$ Individuals may adopt a more normal gait pattern when using trekking poles, resulting in decreased forces on the lower extremities, thus decreasing lower extremity muscle activity. ${ }^{4}$

The literature has shown that use of trekking poles significantly increases upper extremity muscle activity, while potentially decreasing lower extremity muscle activity, particularly when carrying a load. ${ }^{4,13,15}$ This suggests that use of trekking poles offers the benefit of upper extremity exercise, although it may put the user at increased risk of upper extremity muscle strain. Furthermore, for an individual carrying a large external load, the use of trekking poles may be advantageous because the load may be transferred to the poles, resulting in increased efficiency and decreased lower extremity muscle activity.
Table 4. Summary of electromyographic responses of the upper and lower extremities to using trekking poles compared to free walking

\begin{tabular}{|c|c|c|}
\hline Electromyography & $\begin{array}{l}\text { Upper } \\
\text { extremity }\end{array}$ & $\begin{array}{l}\text { Lower } \\
\text { extremity }\end{array}$ \\
\hline Schwameder et al, $1999^{11}$ & - & $\downarrow$ \\
\hline Knight and Caldwell, $2000^{4}$ & - & $\downarrow$ \\
\hline Abendroth-Smith et al, $2008^{9}$ & - & $\uparrow$ \\
\hline Shim et al, $2013^{15}$ & $\uparrow$ & $\leftrightarrow$ \\
\hline Pellegrini et al, $2015^{13}$ & $\uparrow$ & $\leftrightarrow$ \\
\hline
\end{tabular}

\section{BALANCE}

Anecdotal claims commonly suggest poles increase balance and stability during outdoor activities. However, the International Mountaineering and Climbing Federation states that continuous pole use may have an adverse effect on acute balance (ie, if a hiker puts the poles away, balance may be worse than if the hiker had not used poles at all). ${ }^{9}$ The effects of continuous trekking pole use on acute static and dynamic balance have been investigated by several authors (Table 5) ${ }^{9,}{ }^{10}$ In one research study, hikers completed baseline static (standing on a foam pad) and dynamic (walking across a simulated $3.3-\mathrm{m} \log$ ) balance tasks, walked for $15 \mathrm{~min}$ with or without trekking poles, and then repeated the balance task. There were no differences in acute balance between the pole and no-pole condition; although there were fewer balance errors in the pole condition, the difference was not significant. It was concluded that use of poles does not have a negative effect on balance. $^{9}$

Similarly, the effects of poles on balance with and without a load were examined in 3 different conditions: 1) no poles, 2) 1 pole, or 3$) 2$ poles. ${ }^{10}$ In the load condition, participants' balance improved with 2 poles compared to the no-pole condition. In the unloaded condition, balance improved with either 1 or 2 poles compared to the nopole condition, with no differences found between 1 and 2 poles. ${ }^{10}$ These improvements in balance with 1 or 2 poles in loaded and unloaded conditions may reduce a user's risk of falls or injury when trekking. ${ }^{10}$ Thus, based on this study, pole use would be recommended for balance; however, further research is warranted on the effect of single versus double pole use on RPE, EE, and other variables.

Previous research supports claims that use of trekking poles improves acute balance and stability during outdoor activities, ${ }^{9,10}$ but research has yet to investigate the effect of chronic pole use on balance and stability. Nevertheless, the insight provided regarding the effect of pole use on acute balance may be beneficial for those who occasionally use poles during trekking (ie, only when carrying an external load), rather than those who frequently use poles (ie, 
Table 5. Summary of balance responses to various trekking pole conditions compared to no-pole conditions

\begin{tabular}{lcccc}
\hline Balance & Static & Dynamic & Single pole & Double pole \\
\hline Jacobson et al, $1997^{10}$ & - & - & $\uparrow$ & $\uparrow$ \\
Abendroth-Smith et al, $2008^{9}$ & $\uparrow$ & $\uparrow$ & - & - \\
\hline
\end{tabular}

during all outdoor excursions). More research should be conducted on the effects of chronic pole usage on balance.

\section{SPECIAL POPULATIONS' RESPONSE TO USE OF TREKKING POLES}

According to the American College of Sports Medicine, 3 to $5 \mathrm{~d}$ of moderate-intensity aerobic exercise per week is recommended for special populations, including those with Parkinson's disease, obese and elderly people, and those with low back pain. ${ }^{22}$ Types of exercise recommended by the college are those that include prolonged, rhythmic activities using large muscle groups, such as walking, cycling, or swimming. ${ }^{22}$ Another exercise suitable for special populations is Nordic walking (ie, walking with trekking poles), which is an attractive, safe, and accessible mode of physical activity. Nordic walking has been widely studied and has been shown to have a positive impact on mobility, locomotor function, and rehabilitation in the aforementioned special populations. ${ }^{18,23-25}$

Parkinson's disease causes numerous motor deficits (eg, resting tremors, reduced mobility, and stiffness disorders) that may limit functional activities of daily living. ${ }^{26}$ Aerobic exercise may reduce the harmful effects of Parkinson's disease by slowing disease progression, decreasing neuronal death, enhancing cell survival, and stimulating neurogenesis. ${ }^{27}$ Although aerobic exercise is highly recommended, it may not be feasible for those with Parkinson's disease because of the balance and coordination required to complete the activity. The effects of trekking poles and free walking on functional parameters, speed, and locomotor rehabilitation index in patients with Parkinson's disease have been investigated. ${ }^{25,28,29}$ Trekking pole use facilitated better coordination, balance, and functional mobility compared to free walking; it also improved walking speed and timed walking distance. ${ }^{25,28,29}$ Individuals may benefit from using trekking poles because it is a more conscious way of walking; that is, users deliberately use their arms to walk and pay specific attention to their gait. ${ }^{28}$ Use of trekking poles also improved stride length, maximal walking speed, postural stability, and exercise capacity, further supporting that trekking pole use is a beneficial and valuable mode of exercise for those with Parkinson's disease. ${ }^{25,28,29}$ Furthermore, improvements in quality-oflife functional parameters (ie, decreased $10-\mathrm{m}$ walk time and timed get-up-and-go; increased 6-min walk test distance) have been maintained for 5 mo postintervention. ${ }^{28}$ However, it is unknown whether patients continued the Nordic walking program after the study or whether these outcome measures were a result of lifestyle changes. ${ }^{28}$

Physical activity programs are popular among the elderly, likely owing to interest in maintaining and improving physical activity and function, especially as life expectancy continues to rise. ${ }^{24}$ Trekking pole use is recommended for the elderly because of the increased upper extremity recruitment and EE, as well as increased balance and coordination. Few studies have compared the effects of use of trekking poles to free walking or general exercise in elderly populations (60 y or older). Using an 8 - $\mathrm{wk}^{24}$ or 12 - $\mathrm{wk}^{30}$ intervention of 60 -min exercise sessions 3 times each week, gait parameters, locomotor rehabilitation index, quality of life, static balance, and lower and upper extremity strength were examined. Use of trekking poles increased walking speed, balance, and quality of life. However, many of these benefits were observed in free walking as well, suggesting that general physical activity elicits health benefits compared to a sedentary lifestyle. ${ }^{24}$

Similar to the previous studies, the effects of trekking pole use were investigated by comparing elderly adults who regularly walked to sedentary adults and healthy young adults. ${ }^{31}$ Similarities were found in gait between young adults and those who regularly used trekking poles, leading to the conclusion that engaging in physical activity, specifically walking with trekking poles, may counteract gait deterioration that comes with aging. ${ }^{31}$

Because of its accessibility, popularity, and safety, walking is one of the most recommended modes of exercise for the overweight or obese. ${ }^{32}$ However, walking over rough terrain may increase a person's risk of injury and induce fatigue, lower extremity muscle damage, and joint pain. ${ }^{8}$ Over time, this increased loading and stress, specifically at the knee, may lead to cartilage degradation, increasing pain at the joint. ${ }^{23}$ As previously mentioned, trekking poles may reduce lower extremity joint loading, which may slow the degradation and damage of joint cartilage. ${ }^{23}$

The use of trekking poles on muscle and cartilage damage (assessed via blood enzymes) and fatigue during walking at a decline of $15 \%$ was investigated in obese individuals, where participants walked with and without trekking poles. ${ }^{23}$ Immediately after walking with trekking poles, cartilage oligometric matrix protein (COMP) levels 
in the pole condition were lower than the no-pole condition. ${ }^{23}$ Although levels in the no-pole condition were still elevated, COMP levels in the pole condition returned to baseline levels $2 \mathrm{~h}$ postwalking. Because increases in COMP levels after exercise may serve as a biomarker of cartilage degradation and damage, ${ }^{33}$ these findings suggest that using trekking poles may alleviate the temporary muscle and cartilage damage induced after downhill walking. ${ }^{23}$

Low back pain is estimated to affect about $80 \%$ of adults in the United States. ${ }^{34}$ Over time, regular or free walking may aid in reducing the discomfort of those with chronic low back pain. ${ }^{18,35}$ Trekking poles have been shown to alleviate lower extremity joint loading, and they decrease loading of the spine, which in turn may reduce low back pain.

The acute effects of trekking pole use were studied in individuals with mild to moderate low back pain. ${ }^{18}$ After a 5-min warmup with trekking poles, participants walked $0.8 \mathrm{~km}$ with or without poles, rested, and repeated the walk in the opposite condition. Pain, RPE, and HR were monitored pre- and posttask. Low back pain decreased pre- to posttask. Furthermore, pain did not increase after the walking task in either group, leading to the conclusion that using trekking poles when walking may offer some acute benefits and was well tolerated by participants, but use cannot be recommended as a superior method of addressing acute symptoms when walking. ${ }^{18}$

Similarly, individuals with chronic lower back pain were randomly assigned to 3 groups: 1) supervised and structured Nordic walking, 2) $1 \mathrm{~h}$ of Nordic walking instruction, and 3) verbal information about active living and exercise. ${ }^{35}$ After $8 \mathrm{wk}$, there were no differences in low back pain rating, patient-specific function scale, or quality of life among groups. Despite the lack of difference among the groups, the greatest average improvements tended to be in favor of those in the structured Nordic walking group compared to the other 2 groups. ${ }^{35}$

The use of trekking poles during outdoor exercise may be beneficial for those with Parkinson's disease, elderly and obese populations, and those with low back pain. Pole use increased coordination, balance, functional mobility, and quality of life and temporarily alleviated low back pain. ${ }^{18,24,30,35}$ Furthermore, improvements were retained for up to 5 mo after a Nordic walking intervention, which may be beneficial because patients may be able to complete a brief intervention and continue to maintain improvements after the intervention. ${ }^{29}$

\section{Conclusions}

Previous literature has illustrated that using trekking poles increases physiologic responses $\left(\dot{\mathrm{V}}_{2}, \mathrm{HR}\right.$, ventilation, EE) while decreasing biomechanical responses (GRF, joint loading, joint moments) and subjective responses (RPE, DOMS). During free, unloaded walking, users should compare the cost versus benefit of using trekking poles. Although using poles decreases lower extremity loading and forces, cardiovascular demand is increased; thus, a decision should be made based on the individual's current fitness level and whether any lower extremity conditions or pain are present. When carrying a large external load, the use of trekking poles may be beneficial, resulting in decreased lower extremity muscle activity and increased balance and stability, with no meaningful differences in metabolic expenditure. Future research should consider investigating the effect of trekking poles on lower extremity $\mathrm{EE}$, as well as the effect of single versus double pole use on RPE and EE.

Author Contributions: Study concept and design (ALH); acquisition of the data (ALH); analysis of the data (ALH); drafting of the manuscript (ALH, RLJ); critical revision of the manuscript (ALH, RLJ); approval of the final manuscript (ALH, RLJ).

Financial/Material Support: None.

Disclosures: None.

\section{References}

1. Mitten D, Overholt JR, Haynes FI, D’Amore CC, Ady JC. Hiking: a low-cost, accessible intervention to promote health benefits. Am J Lifestyle Med. 2016;12(4):302-10.

2. Lobb B. Load carriage for fun: a survey of New Zealand trampers, their activities and injuries. Appl Ergon. 2004;35(6): $541-7$.

3. Frederick EC. Estimates of the energy cost of rifle carriage in biathlon ski skating. J Appl Biomech. 1987;3(4):392-403.

4. Knight CA, Caldwell GE. Muscular and metabolic costs of uphill backpacking: are hiking poles beneficial? Med Sci Sports Exerc. 2000;32(12):2093-101.

5. Svensson M. Nordic walking. In: Steffen J, Stiehl J, eds. Teaching Lifetime Outdoor Pursuits. Champaign, IL: Human Kinetics; 2010:191-205.

6. Bohne M, Abendroth-Smith J. Effects of hiking downhill using trekking poles while carrying external loads. Med Sci Sports Exerc. 2007;39(1):177-83.

7. Park SK, Yang DJ, Kang YH, Kim JH, Uhm YH, Lee YS. Effects of Nordic walking and walking on spatiotemporal gait parameters and ground reaction force. J Phys Ther Sci. 2015;27(9):2891-3.

8. Howatson G, Hough P, Pattison J, Hill JA, Blagrove R, Glaister M, et al. Trekking poles reduce exercise-induced muscle injury during mountain walking. Med Sci Sports Exerc. 2011;43(1):140-5.

9. Abendroth-Smith J, Swigart V, Dixon G, Bohne M. The effects of continuous hiking pole use on acute static and dynamic balance of men and women hikers. Med Sci Sports Exerc. 2009;41(5):510.

10. Jacobson BH, Caldwell B, Kulling FA. Comparison of hiking stick use on lateral stability while balancing with and without a load. Percept Mot Skills. 1997;85(1):347-50. 
11. Schwameder H, Roithner R, Muller E, Niessen W, Raschner C. Knee joint forces during downhill walking with hiking poles. J Sports Sci. 1999;17(12):969-78.

12. Hansen EA, Smith G. Energy expenditure and comfort during Nordic walking with different pole lengths. J Strength Cond Res. 2009;23(4):1187-94.

13. Pellegrini B, Peyré-Tartaruga LA, Zoppirolli C, Bortolan L, Bacchi E, Figard-Fabre H, et al. Exploring muscle activation during Nordic walking: A comparison between conventional and uphill walking. PLOS ONE. 2015;10(9):e0138906.

14. Grainer A, Zerbini L, Reggiani C, Marcolin G, Steele J, Pavei G, et al. Physiological and perceptual responses to Nordic walking in a natural mountain environment. Int J Environ Res Public Health. 2017;14(10):1235.

15. Shim J, Kwon H, Kim H, Kim B, Jung J. Comparison of the effects of walking with and without Nordic pole on upper extremity and lower extremity muscle activation. J Phys Ther Sci. 2013;25(12):1553-6.

16. Saunders MJ, Hipp GR, Wenos DL, Deaton ML. Trekking poles increase physiological responses to hiking without increased perceived exertion. J Strength Cond Res. 2008;22(5):1468-74.

17. Schiffer T, Knicker A, Dannöhl R, Strüder HK. Energy cost and pole forces during Nordic walking under different surface conditions. Med Sci Sports Exerc. 2009;41(3):663-8.

18. Revord LP, Lomond KV, Loubert PV, Hammer RL. Acute effects of walking with Nordic poles in persons with mild to moderate low-back pain. Int J Exerc Sci. 2016;9(4):507-13.

19. Willson J, Torry MR, Decker MJ, Kernozek T, Steadman JR. Effects of walking poles on lower extremity gait mechanics. Med Sci Sports Exerc. 2001;33(1):142-7.

20. Alexander N, Schwameder H. Lower limb joint forces during walking on the level and slopes at different inclinations. Gait Posture. 2016;45:137-42.

21. Kuster M, Sakurai S, Wood GA. Kinematic and kinetic comparison of downhill and level walking. Clin Biomech (Bristol, Avon). 1995;10(2):79-84.

22. ACSM's Guidelines for Exercise Testing and Prescription. 10th ed. Wolters Kluwer; 2018.

23. Cho SY, Roh HT. Trekking poles reduce downhill walkinginduced muscle and cartilage damage in obese women. J Phys Ther Sci. 2016;28(5):1574-6.
24. Gomeñuka NA, Oliveira HB, Silva ES, Costa RR, Kanitz AC, Liedtke GV, et al. Effects of Nordic walking training on quality of life, balance and functional mobility in elderly: a randomized clinical trial. PLOS ONE. 2019;14(1), e0211472.

25. Monteiro EP, Franzoni LT, Cubillos DM, de Oliveira Fagundes A, Carvalho AR, Oliveira HB, et al. Effects of Nordic walking training on functional parameters in Parkinson's disease: A randomized controlled clinical trial. Scand J Med Sci Sports. 2017;27(3):351-8.

26. Kluger BM, Brown RP, Aerts S, Schenkman M. Determinants of objectively measured physical functional performance in early to mid-stage Parkinson disease. PM R. 2014;6(11):992-8.

27. Frazzitta G, Balbi P, Maestri R, Bertotti G, Boveri N, Pezzoli G. The beneficial role of intensive exercise on Parkinson disease progression. Am J Phys Med Rehabil. 2013;92(6): $523-32$.

28. van Eijkeren FJM, Reijmers RSJ, Kleinveld MJ, Minten A, Bruggen JPT, Bloem BR. Nordic walking improves mobility in Parkinson's disease. Mov Disord. 2008;23(15):2239-43.

29. Reuter I, Mehnert S, Leone P, Kaps M, Oechsner M, Engelhardt M. Effects of a flexibility and relaxation programme, walking, and Nordic walking on Parkinson's disease. J Aging Res. 2011;2011:232473.

30. Lee HS, Park JH. Effects of Nordic walking on physical functions and depression in frail people aged 70 years and above. J Phys Ther Sci. 2015;27(8):2453-6.

31. Mansour KB, Gorce P, Rezzoug N. The impact of Nordic walking training on the gait of the elderly. J Sports Sci. 2018;36(20):2368-74.

32. Poirier P, Després JP. Exercise in weight management of obesity. Cardiol Clin. 2001;19(3):459-70.

33. Vuolteenaho K, Leppänen T, Kekkonen R, Korpela R, Moilanen E. Running a marathon induces changes in adipokine levels and in markers of cartilage degradation-novel role for resistin. PLOS ONE. 2014;9(10):e110481.

34. Andersson GB. Epidemiological features of chronic low-back pain. Lancet. 1999;354(9178):581-5.

35. Hartvigsen J, Mors $\varnothing$ L, Bendix T, Manniche C. Supervised and non-supervised Nordic walking in the treatment of chronic low back pain: a single blind randomized clinical trial. BMC Musculoskelet Disord. 2010;11:30. 